\title{
Electrodeposition of Ni micro/nano-structures
}

\author{
NIU LianPing ${ }^{1 *}$, YE DanDan ${ }^{1}$, LI Feng $^{1} \&$ ZHANG MingZhe ${ }^{2}$ \\ ${ }^{1}$ College of Mathematics, Physics and Information Engineering, Jiaxing University, Jiaxing 314001, China; \\ ${ }^{2}$ National Laboratory of Superhard Materials and Institute of Atomic and Molecular Physics, Jilin University, Changchun 130012, China
}

Received May 23, 2011; accepted June 28, 2011

A quasi two-dimension electrodeposition method was used to prepare materials with arborized, tree-shaped, and membrane patterned micro/nano-structures. The morphology of the membrane patterned micro/nano-structure was characterized using scanning electron microscopy, and a mechanism for the morphology formation was suggested. The novel preparation method used in this study, which is proprietary to our research group, does not require a template and so has many advantages including controllable, low cost, large scale preparation. The electrolyte concentration and electrodeposition voltage had a significant effect on the resulting morphology.

Ni electrodeposition, micro/nano-structures, electric potential concentration

Citation: $\quad$ Niu L P, Ye D D, Li F, et al. Electrodeposition of Ni micro/nano-structures. Chinese Sci Bull, 2011, 56: 3426-3430, doi: 10.1007/s11434-011-4766-7

Micro/nano-structures have attracted much recent attention because of their electrical [1], optical [2], chemical [3], catalysis [4] and magnetic [5,6] properties. They have potential applications in micro/nano-electronic devices, sensors and high density magnetic recording devices [7-10]. Ni is a useful magnetic material with unique properties. The highly ordered magnetic structure of $\mathrm{Ni}$ can induce outstanding cooperative phenomena that differ from the bulk and thin film analogs, and Ni micro/nano-structures have been extensively investigated [11]. Much research has focused on studying the characteristics and applications of $\mathrm{Ni}$ micro/nano-structures [12-20]. However, for such devices to be designed and applied, Ni micro/nano-structured materials must first be prepared. Vazquez et al. [21] fabricated arrays of $\mathrm{Ni}$ nanowires in alumina membranes, and studied the magnetic properties and interactions which influenced spatial ordering. Ohgai et al. [22] also studied Ni nanostructures, in which a polycarbonate film was irradiated with high energetic heavy ion beams to form straight tracks perpendicular to the film. Films were subsequently selectively etched to create cylindrical nanochannels where $\mathrm{Ni}$ nan-

*Corresponding author (email: nlpxy@126.com) owires were electrodeposited, and their anisotropic magnetoresistance (AMR) behavior was investigated. Narayanan et al. [23] fabricated Ni nanowires using template assisted electrodeposition, and studied the nanowire growth mechanism and magnetization behavior. Bentley et al. [24] prepared $\mathrm{Ni}$ nanowires using a template synthesis technique and characterized the nanowire properties. The alignment and movement of the nanowires could be controlled magnetically. Liu et al. [25] prepared Ni nanowires without a template using a magnetic field. Wang et al. [26] prepared Ni nanowires by a soft template method in ethylene glycol. Most of these reports involve templates being introduced to fabricate micro/nano-structures. While this method is very effective and the size and ordering can be controlled, templates must first be made from complicated and costly procedures.

In the current paper, chemical electrodeposition [27-33] without templates is used to prepare micro/nano-structures. Under an applied external voltage, solution phase metal ions are reduced to form deposited layers. When a voltage is applied across an electrode, metal atoms of the anode lose electrons to become cations, which then proceed into the electrolyte solution. Cations in the electrolyte move towards 
the cathode due to the electric field, where they then gain electrons. Lost electrons flow out of the anode and proceed back to the positive electrode via the electrical circuit, and then proceed to the cathode. A current circuit is formed depending on the movement of cations and electrons, and the metallic material can then be electrodeposited. Micro/nanostructure electrodeposition generally includes two processes, one forming the crystal nucleus and the other being the growth of that nucleus.

\section{Materials and methods}

$\mathrm{NiCl}_{2}$ solutions with concentrations of $10,30,40,50$ and $80 \mathrm{mmol} / \mathrm{L}$ were prepared. Two Ni electrodes were fabricated, each being $30 \mathrm{~mm}$ long, $5 \mathrm{~mm}$ wide and $0.07 \mathrm{~mm}$ thick. Two Ni electrodes with wires were placed parallel to each other on $5 \mathrm{~cm} \times 5 \mathrm{~cm}$ clean silicon substrate. Two or three drops of $\mathrm{NiCl}_{2}$ solution were placed on the silicon substrate between the two electrodes, and with the electrodes and solution; then covered with a glass slide. The distance between the two electrodes was approximately $2 \mathrm{~cm}$. Figure 1 shows the system comprised of electrodes, silicon substrate and electrolyte solution.

The next step was to fabricate an ultrathin liquid layer of $\mathrm{NiCl}_{2}$. According to the principle of solute respective solidification, when the temperature decreases, the $\mathrm{NiCl}_{2}$ electrolyte solution will freeze. The $\mathrm{NiCl}_{2}$ solute would then separate from solution and reside between the ice and $\mathrm{Si}$ substrate layers, thus forming an ultrathin solute layer. Thus, the $\mathrm{NiCl}_{2}$ solution was effectively localized into a two dimensional field, and when deposits grew in a single plane then ordered structures could be prepared. The power source was supplied by a function signal generator, with a constant voltage used to drive the electrodeposition and initiate the material preparation. Material growth was monitored using an optical microscope. The voltage was adjusted as necessary to control the growth speed and morphology.

\section{Results and discussion}

\subsection{Characterization of arborized and tree-shaped $\mathrm{Ni}$ micro/nano-structures}

Three different shaped micro/nano-structures were obtained from the electrodeposition experiments. Figure 2 shows

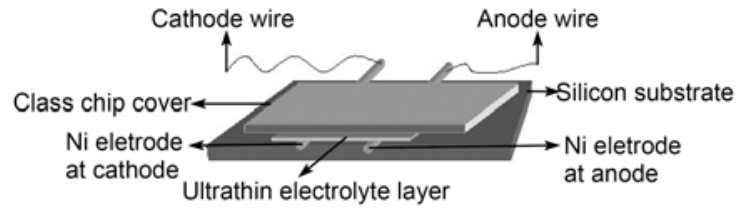

Figure 1 Schematic diagram of the preparation equipment comprised of electrodes, silicon substrate and electrolyte solution.
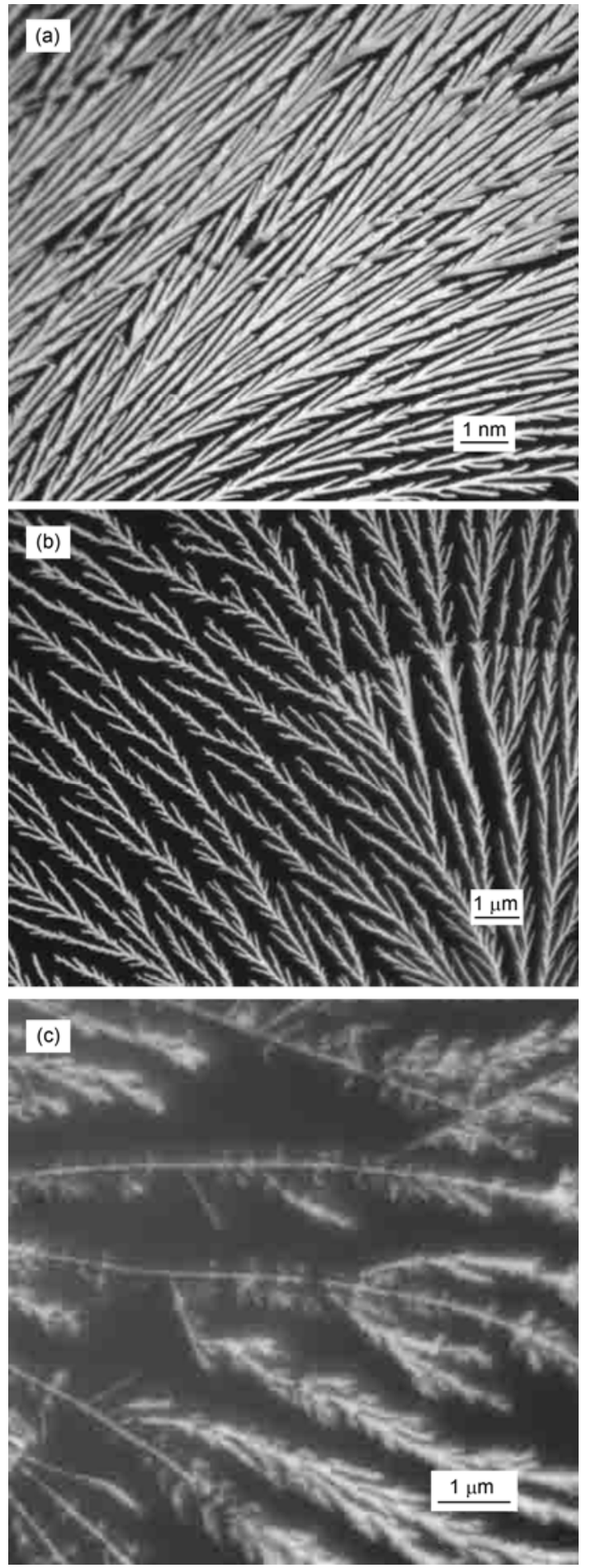

Figure 2 Optical microscope images of arborized Ni micro/nano-structures. Electrolyte concentration, applied voltage and $\mathrm{pH}$ were: (a) $10 \mathrm{mmol} / \mathrm{L}, 1.1$ $\mathrm{V}$, and 1.95; (b) $30 \mathrm{mmol} / \mathrm{L}, 1.4 \mathrm{~V}$, and 1.90; (c) $40 \mathrm{mmol} / \mathrm{L}, 1.5 \mathrm{~V}$, and 1.80 .

arborized structures, of which three types were observed. The first type was distributed densely and single 'trees' contained few bifurcations, as shown in Figure 2(a). The second was distributed more sparsely but single 'trees' contained many bifurcations, as shown in Figure 2(b). The third 
was distributed very sparsely and single 'trees' were more spinous, as shown in Figure 2(c). These results show that when the electrodeposition voltage and electrolyte concentration were higher, the resulting micro/nano-structures were more bifurcated. Overall, micro/nano-structures of sparse or linear arrays were easily formed. The thickness of the ultrathin liquid layer can also affect resulting morphology, because a thinner layer leads to more regular and wellproportioned deposition.

Tree-shaped Ni micro/nano-structures are shown in Figure 3 , in which the trunks are straight. While individual 'trees' were sparse, single 'trees' exhibited many forks.

A mechanism for the electrodeposition procedure giving the above morphologies is suggested below. A lower concentration electrolyte was used to prepare the above samples. During electrodeposition, electrons were distributed from the head of the Ni sample. When electron distribution was constant thus achieving an equilibrium supply and consumption, Ni deposition did become bifurcated.

When the applied voltage was increased, the equilibrium between the supply and consumption of electrons was perturbed, leading to an increase in over potential and increase in deposition rate. If electrons were still in surplus in addition to consumed electron due to growth, electrons in the head of the deposition would continue to accumulate with further growth, and when this accumulation reached a certain level then repulsion between electrons would occur. In this situation, electron distribution would no longer be equal. Electrons that were confined at the head of the deposit would move toward the alternative end to minimize this repulsion. Two electron areas were formed, and when transported $\mathrm{Ni}^{2+}$ arrived at these areas, $\mathrm{Ni}^{2+}$ was reduced and bifurcation occurred as shown in Figure 4.

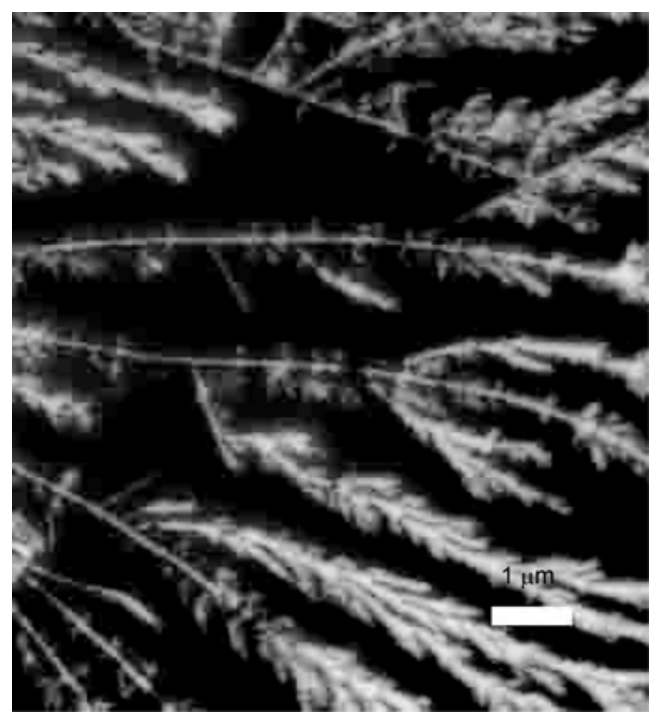

Figure 3 Optical microscope image of a tree-shaped Ni micro/nano-structure. Electrolyte concentration, applied voltage and $\mathrm{pH}$ were $50 \mathrm{mmol} / \mathrm{L}, 1.8 \mathrm{~V}$ and 1.62 , respectively.

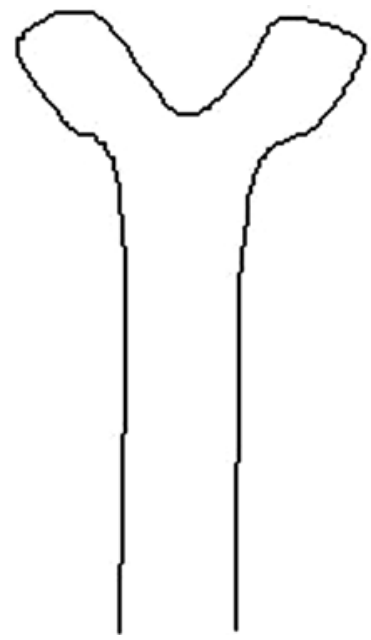

Figure 4 Schematic diagram showing bifurcation of a deposit.

If the applied voltage was lower, surplus electron density was lower and electron repulsion was weaker. Thus, the distance between electrons at the head of the deposit was less and growth was slower. The deposit bifurcated to a lesser degree giving a denser deposit, as shown in Figure 2(a). If the applied voltage was increased, surplus electron density was greater and electron repulsion was stronger. The distance between electrons was greater and the growth faster quicker. The deposit bifurcated to a greater degree giving a more sparse deposit, as shown in Figure 2(b). When the applied voltage was significantly increased, surplus electron density was very high and electron repulsion was very strong. The distance between electrons was very large and the growth was rapid. The deposit bifurcated to a very high degree, as shown in Figure 2(c). The different micro/nano-structures reported above are important for understanding the mechanism of crystal growth and also the possible uses of the materials. Arborized structures are more ordered and can be made over a larger area, thus could be applied in ultrahigh density magnetic storage devices. The recoding densities of such devices will be much higher than those obtained from conventional continuous magnetic films.

\subsection{Characterization of membrane patterned Ni micro/ nano-structure}

The membrane patterned Ni micro/nano-structure is shown in Figure 5. Figures 5(a) and (b) are images of a single sample but at different magnifications. Arrows indicate the growth orientation of the material which is aligned with the applied electric field. Figure 5(a) shows an obvious periodic morphology and a smooth membrane surface. Figure 4(b) shows that the membrane had a vivid interface between periods, and that the period length was $\sim 2 \mu \mathrm{m}$.

The membrane was easily prepared by electrodeposition under the conditions of high electrolyte concentration 

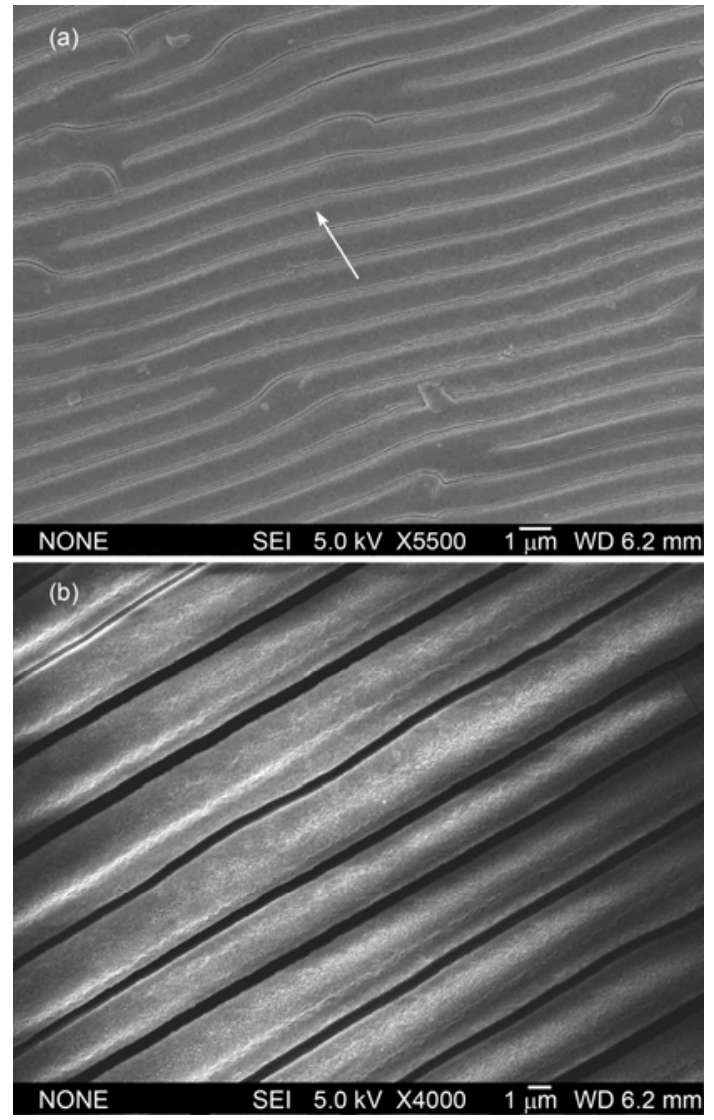

Figure 5 SEM images of the membrane patterned Ni micro/nano-structure. Electrolyte concentration, applied voltage and $\mathrm{pH}$ were $80 \mathrm{mmol} / \mathrm{L}, 4.0 \mathrm{~V}$ and 1.53 , respectively.

( $>50 \mathrm{mmol} / \mathrm{L}$ ) and voltage $(>4 \mathrm{~V}$ ), but the growth process was slow. In preparing $\mathrm{Ni}$ micro/nano-structures, the predominant chemical reaction is the reduction of $\mathrm{Ni}^{2+}$ to $\mathrm{Ni}$ at the cathode. The reaction equation is $\mathrm{Ni}^{2+}+2 \mathrm{e}^{-}=\mathrm{Ni}$. Reduced $\mathrm{Ni}^{2+}$ initially formed crystal nuclei and those nuclei grew into the above $\mathrm{Ni}$ micro/nano-structures.

A mechanism for the electrodeposition of the membrane is suggested below. A high concentration electrolyte was used, and the applied potential corresponded to the electrolyte concentration. During electrodeposition, a small potential relative to the electrolyte concentration resulted in a slow growth speed. Electrons had sufficient time to migrate upon repulsion, in the direction parallel to the electric field, and thus were uniformly distributed in one plane. The distance between two neighboring electrons was relatively close following their rearrangement for repulsion. When $\mathrm{Ni}^{2+}$ arrived at the surface, $\mathrm{Ni}^{2+}$ was reduced and the $\mathrm{Ni}$ structure grew. If electrons were stoichiometrically consumed during reaction then there was no surplus electron density. The transportation of $\mathrm{Ni}^{2+}$ and consumption of electrons by reduction achieved equilibrium, resulting in deposition of a membrane structure but without a period. If electrons were in surplus, then the potential of the cathode would become more negative. The over potential of the cathode would increase, thus the speed of transportation and reduction of $\mathrm{Ni}^{2+}$ would also increase leading to electron depletion at the cathode. Reduction ceased at the cathode when the over potential decreased to zero. When the cathode become charged following the arrival of $\mathrm{Ni}^{2+}$, the electrode potential increased, the cathode over potential was again present, and thus reduction occurred again. The process repeated to form an undulated structure with over potential. When over potential was present the reaction proceeded. When over potential was lower than the reduction potential the reaction paused. The resulting membrane had a periodic structure as shown in Figure 5.

\section{Conclusions}

Three different $\mathrm{Ni}$ micro/nano-structures were prepared. The morphology of the resulting structures could be controlled by adjusting the electrodeposition conditions. It was easier to fabricate ordered patterns at lower applied voltages and electrolyte concentrations, which is important for the electrodeposition process. Our results provided theory related to chemical electrodeposition supported by experimental data, and will be important for the future development of micro/nano-structures materials. The prepared structures had unusual morphologies that have potential applications in micro/nano-devices.

This work was supported by the National Natural Science Foundation of China (50672029, 90923032 and 20873052). The authors thank Prof. Zhang Mingzhe and Dr. Zong Zhaocun for helpful discussions and review of this paper.

1 Link S, El-Sayed M A. Optical properties and ultrafast dynamics of metallic nanocrystals. Annu Rev Phys Chem, 2003, 54: 331-366

2 Lu A H, Salabas E L, Schüth F. Magnetic nanoparticles: Synthesis, protection, functionalization, and application. Angew Chem Int Ed, 2007, 46: 1222-1244

3 Braun E, Eichen Y, Sivan U, et al. DNA templated assembly and electrode attachment of a conducting silver wire. Nature, 1998, 391: $775-778$

4 Narayanan R, El-Sayed M A. Catalysis with transition metal nanoparticles in colloidal solution: Nanoparticle shape dependence and stability. J Phys Chem B, 2005, 109: 12663-12676

5 Kovtyukhova N I, Mallouk T E. Nanowires as building blocks for self-assembling logic and memory circuits. Chem Eur J, 2002, 8: 4354-4363

6 Patolsky F, Zheng G, Lieber C M, et al. Electrical detection of single viruses. Proc Natl Acad Sci USA, 2004, 104: 14017-14022

7 Gudiksen M S, Lauhon L J, Wang J F, et al. Growth of nanowire superlattice structures for nanoscale photonics and electronics. Nature, 2002, 415: 617-620

8 Cui Y, Wei Q Q, Park H K, et al. Nanowire nanosensors forhighly sensitive and selective detection of biological and chemical species. Science, 2001, 293: 1289-1292

9 Thurn-Albrecht T, Schotter J, Kastle G A, et al. Ultrahigh-density nanowire arrays grown in self-assembled diblock copolymer templates. Science, 2000, 290: 2126-2129

10 Liang H P, Guo Y Q, Hu J S, et al. Ni-Pt multilayered nanowire arrays with enhanced coercivity and high remanence ratio. Inorg Chem, 
2005, 44: 3013-3015

11 Pirota K R, Navas D, Hernandez-Velez M, et al. Novel magnetic materials prepared by electrodeposition techniques: Arrays of nanowires and multi-layered microwires. J Alloy Compd, 2004, 369: 18-26

12 Tian F, Zhu J, Wei D. Fabrication and magnetism of radial-easy-magnetized Ni nanowire arrays. J Phys Chem C, 2007, 111: 12669-12673

13 Hangarter C M, Myung N V. Magnetic alignment of nanowires. Chem Mater, 2005, 17: 1320-1324

14 Shi J B, Chen Y C, Lee C W, et al. Optical and magnetic properties of 30 and $60 \mathrm{~nm}$ Ni nanowires. J Mater Lett, 2008, 62: 15-18

15 Pan H, Liu B H, Yi J B, et al. Growth of single-crystalline Ni and Co nanowires via electrochemical deposition and their magnetic properties. J Phys Chem B, 2005, 109: 3094-3098

16 Jin C G, Liu W F, Jia C, et al. High-filling, large-area Ni nanowire arrays and the magnetic properties. J Cryst Growth, 2003, 258: 337-341

17 Sellmyer D J, Zheng M, Skomski R. Magnetism of Fe, Co and $\mathrm{Ni}$ nanowires in self-assembled arrays. J Phys Condensed Mater, 2001, 13: R433

18 Tian F, Chen J, Zhu J. Magnetism of thin polycrystalline nickel nanowires. J Apply Phys, 2008, 103: 013901

19 Cao H Q, Wang L D, Qiu Y, et al. Generation and growth mechanism of metal (Fe, Co, Ni) nanotube arrays. ChemPhysChem, 2006, 7: $1500-1504$

20 Wang Q T, Wang G Z, Han X H, et al. Controllable template synthesis of $\mathrm{Ni} / \mathrm{Cu}$ nanocable and $\mathrm{Ni}$ nanotube arrays: A one-step coelectrodeposition and electrochemical etching method. J Phys Chem B, 2005, 109: 23326-23329

21 Vazquez M, Hernandez-Velez M, Pirota K, et al. Arrays of Ni nanowires in alumina membranes: Magnetic properties and spatial ordering. Eur Phys J B, 2004, 40: 489-497

22 Ohgai T, Enculesu I, Zet C, et al. Magneto-sensitive nickel nanowires fabricated by electrodeposition into multi- and single-ion track templates. J Appl Elecrtrochem, 2006, 36: 1157-1162

23 Narayanan T N, Shaijumon M M, Ci L J, et al. On the growth mechanism of nickel and 24 cobalt nanowires and comparison of their magnetic properties. Nano Res, 2008, 1: 465-473

24 Bentley A K, Farhoud M, Arthur B E. Template synthesis and magnetic manipulation of nickel nanowires. J Chem Educ, 2005, 82: 765

25 Liu P, Li Z J, Zhao B, et al. Template-free synthesis of nickel nanowires by magnetic field. Mater Lett, 2009, 63: 1650-1652

26 Wang D P, Sun D B, Yu H Y, et al. Preparation of one-dimensional nickel nanowires by self-assembly process. Mater Chem Phys, 2009, 113: 227-232

27 Li X R, Wang Y Q, Song G J, et al. Synthesis and growth mechanism of $\mathrm{Ni}$ nanotubes and nanowires. Nanoscale Res Lett, 2009, 4: $1015-1020$

28 Penner R M, Martin C R. Preparation and electrochemical characterization of ultramicroelectrode ensembles. Anal Chem, 1987, 59: 2625-2630

29 Yin A J, Li J, Jian W, et al. Nonlinear magneto-optical Kerr effect in hyper-Rayleigh scattering from layer-by-layer assembled films of yttrium iron garnet nanoparticles. Appl Phys Lett, 2001, 79: 1039-1044

30 Zhang M Z, Lenhert S, Wang M, et al. Microcontact printing of CdS/dendrimer nanocomposite patterns on silicon wafers. Adv Mater, 2004, 16: 409-413

31 Zhang M Z, Wang M, Zhang Z, et al. Periodic structures of randomly distributed $\mathrm{Cu} / \mathrm{Cu}_{2} \mathrm{O}$ nanograins and periodic variations of cell voltage in copper electrodeposition. Electrochim Acta, 2004, 49: 2379-2383

32 Cheng C D, Ravi K G, Qun G, et al. Self-assembly of metallic nanowires from aqueous solution. Nano Lett, 2005, 5: 175-178

33 Zong Z C, Yu H, Niu L P, et al. Potential-induced copper periodic micro-/nanostructures by electrodeposition on silicon substrate. Nanotechnology, 2008, 19: 315302-315307

Open Access This article is distributed under the terms of the Creative Commons Attribution License which permits any use, distribution, and reproduction in any medium, provided the original author(s) and source are credited. 